\title{
Three Solutions to Dirichlet Boundary Value Problems for $p$-Laplacian Difference Equations
}

\author{
Liqun Jiang ${ }^{1,2}$ and Zhan Zhou' ${ }^{2,3}$ \\ ${ }^{1}$ Department of Mathematics and Computer Science, Jishou University, Jishou, Hunan 416000, China \\ ${ }^{2}$ Department of Applied Mathematics, Hunan University, Changsha, Hunan 410082, China \\ ${ }^{3}$ Department of Applied Mathematics, Guangzhou University, Guangzhou 510006, Guangdong, China
}

Correspondence should be addressed to Liqun Jiang, liqunjianghn@yahoo.com

Received 2 March 2007; Revised 16 July 2007; Accepted 15 October 2007

Recommended by Svatoslav Stanek

We deal with Dirichlet boundary value problems for $p$-Laplacian difference equations depending on a parameter $\lambda$. Under some assumptions, we verify the existence of at least three solutions when $\lambda$ lies in two exactly determined open intervals respectively. Moreover, the norms of these solutions are uniformly bounded in respect to $\lambda$ belonging to one of the two open intervals.

Copyright $@ 2008$ L. Jiang and Z. Zhou. This is an open access article distributed under the Creative Commons Attribution License, which permits unrestricted use, distribution, and reproduction in any medium, provided the original work is properly cited.

\section{Introduction}

Let $\mathbb{R}, \mathbb{Z}, \mathbb{N}$ be all real numbers, integers, and positive integers, respectively. Denote $\mathbb{Z}(a)=$ $\{a, a+1, \ldots\}$ and $\mathbb{Z}(a, b)=\{a, a+1, \ldots, b\}$ with $a<b$ for any $a, b \in \mathbb{Z}$.

In this paper, we consider the following discrete Dirichlet boundary value problems:

$$
\begin{gathered}
\Delta\left[\phi_{p}(\Delta x(k-1))\right]+\lambda f(k, x(k))=0, \quad k \in \mathbb{Z}(1, T), \\
x(0)=0=x(T+1),
\end{gathered}
$$

where $T$ is a positive integer, $p>1$ is a constant, $\Delta$ is the forward difference operator defined by $\Delta x(k)=x(k+1)-x(k), \phi_{p}(s)$ is a $p$-Laplacian operator, that is, $\phi_{p}(s)=|s|^{p-2} s, f(k, \cdot) \in C(\mathbb{R}, \mathbb{R})$ for any $k \in \mathbb{Z}(1, T)$.

There seems to be increasing interest in the existence of solutions to boundary value problems for finite difference equations with $p$-Laplacian operator, because of their applications in many fields. Results on this topic are usually achieved by using various fixed point theorems in cone; see [1-4] and references therein for details. It is well known that critical point theory is an important tool to deal with the problems for differential equations. 
In the last years, a few authors have gradually paid more attentions to applying critical point theory to deal with problems for nonlinear second discrete systems; we refer to [5-9]. But all these systems do not concern with the $p$-Laplacian. For the reader's convenience, we recall the definition of the weak closure.

Suppose that $E \subset X$. We denote $\bar{E}^{w}$ as the weak closure of $E$, that is, $x \in \bar{E}^{w}$ if there exists a sequence $\left\{x_{n}\right\} \subset E$ such that $\Lambda x_{n} \rightarrow \Lambda x$ for every $\Lambda \in X^{*}$.

Very recently, based on a new variational principle of Ricceri [10], the following three critical points was established by Bonanno [11].

Theorem 1.1 (see [11, Theorem 2.1]). Let $X$ be a separable and reflexive real Banach space. $\Phi: X \rightarrow \mathbb{R}$ a nonnegative continuously Gâteaux differentiable and sequentially weakly lower semicontinuous functional whose Gâteaux derivative admits a continuous inverse on $X^{*} . J: X \rightarrow \mathbb{R}$ a continuously Gâteaux differentiable functional whose Gâteaux derivative is compact. Assume that there exists $x_{0} \in X$ such that $\Phi\left(x_{0}\right)=J\left(x_{0}\right)=0$ and that

(i) $\lim _{\|x \rightarrow+\infty\|}(\Phi(x)-\lambda J(x))=+\infty$ for all $\lambda \in[0,+\infty[$;

Further, assume that there are $r>0, x_{1} \in X$ such that

(ii) $r<\Phi\left(x_{1}\right)$;

(iii) $\sup _{x \in \bar{\Phi}^{-1}(]-\infty, r[)} w J(x)<\left(r /\left(r+\Phi\left(x_{1}\right)\right)\right) J\left(x_{1}\right)$.

Then, for each

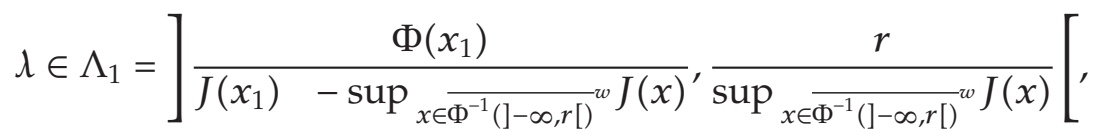

the equation

$$
\Phi^{\prime}(x)-\lambda J^{\prime}(x)=0
$$

has at least three solutions in $X$ and, moreover, for each $h>1$, there exists an open interval

$$
\Lambda_{2} \subseteq\left[0, \frac{h r}{r\left(J\left(x_{1}\right) / \Phi\left(x_{1}\right)\right)-\sup _{x \in \bar{\Phi}^{-1}(]-\infty, r[} w J(x)}\right]
$$

and a positive real number $\sigma$ such that, for each $\lambda \in \Lambda_{2}$, (1.3) has at least three solutions in X whose norms are less than $\sigma$.

Here, our principle aim is by employing Theorem 1.1 to establish the existence of at least three solutions for the $p$-Laplacian discrete boundary value problem (1.1).

The paper is organized as follows. The next section is devoted to give some basic definitions. In Section 3, under suitable hypotheses, we prove that the problem (1.1) possesses at least three solutions when $\lambda$ lies in exactly determined two open intervals, respectively; moreover, all these solutions are uniformly bounded with respect to $\lambda$ belonging to one of the two open intervals. At last, a consequence is presented.

\section{Preliminaries}

The class $H$ of the functions $x: \mathbb{Z}(0, T+1) \rightarrow \mathbb{R}$ such that $x(0)=x(T+1)=0$ is a $T$-dimensional Hilbert space with inner product

$$
(x, z)=\sum_{k=1}^{T} x(k) z(k), \quad \forall x, z \in H
$$


We denote the induced norm by

$$
\|x\|=\left(\sum_{k=1}^{T} x^{2}(k)\right)^{1 / 2}, \quad x \in H .
$$

Furthermore, for any constant $p>1$, we define other norms

$$
\begin{aligned}
& \|x\|_{p}=\left(\sum_{k=1}^{T}|x(k)|^{p}\right)^{1 / p}, \quad \forall x \in H, \\
& \|x\|_{p}=\left(\sum_{k=1}^{T+1}|\Delta x(k-1)|^{p}\right)^{1 / p}, \quad \forall x \in H .
\end{aligned}
$$

Since $H$ is a finite dimensional space, there exist constants $c_{2 p} \geq c_{1 p}>0$ such that

$$
c_{1 p}\|x\|_{p} \leq\|x\|_{P} \leq c_{2 p}\|x\|_{p} .
$$

The following two functionals will be used later:

$$
\Phi(x)=\frac{1}{p} \sum_{k=1}^{T+1}|\Delta x(k-1)|^{p}, \quad J(x)=\sum_{k=1}^{T} F(k, x(k)),
$$

where $x \in H, F(k, \xi):=\int_{0}^{\xi} f(k, s) d s$ for any $\xi \in \mathbb{R}$. Obviously, $\Phi, J \in C^{1}(H, \mathbb{R})$, that is, $\Phi$ and $J$ are continuously Fréchet differentiable in $H$. Using the summation by parts formula and the fact that $x(0)=x(T+1)=0$ for any $x \in H$, we get

$$
\begin{aligned}
\Phi^{\prime}(x)(z) & =\lim _{t \rightarrow 0} \frac{\Phi(x+t z)-\Phi(x)}{t}=\sum_{k=1}^{T+1}|\Delta x(k-1)|^{p-2} \Delta x(k-1) \Delta z(k-1) \\
& =\sum_{k=1}^{T+1} \phi_{p}(\Delta x(k-1)) \Delta z(k-1) \\
& =\sum_{k=1}^{T} \phi_{p}(\Delta x(k-1)) \Delta z(k-1)-\phi_{p}(\Delta x(T)) z(T) \\
& =\left.\phi_{p}(\Delta x(k-1)) z(k-1)\right|_{1} ^{T+1}-\sum_{k=1}^{T} \Delta \phi_{p}(\Delta x(k-1)) z(k)-\phi_{p}(\Delta x(T)) z(T) \\
& =-\sum_{k=1}^{T} \Delta \phi_{p}(\Delta x(k-1)) z(k)
\end{aligned}
$$

for any $x, z \in H$. Noticing the fact that $x(0)=x(T+1)=0$ for any $x \in H$ again, we obtain

$$
J^{\prime}(x)(z)=\lim _{t \rightarrow 0} \frac{J(x+t z)-J(x)}{t}=\sum_{k=1}^{T} f(k, x(k)) z(k)
$$

for any $x, z \in H$. 
Remark 2.1. Obviously, for any $x, z \in H$,

$$
(\Phi-\lambda J)^{\prime}(x)(z)=-\sum_{k=1}^{T}\left[\Delta \phi_{p}(\Delta x(k-1))+\lambda f(k, x(k))\right] z(k)=0
$$

is equivalent to

$$
\Delta \phi_{p}(\Delta x(k-1))+\lambda f(k, x(k))=0
$$

for any $k \in \mathbb{Z}(1, T)$ with $x(0)=x(T+1)=0$. That is, a critical point of the functional $\Phi-\lambda J$ corresponds to a solution of the problem (1.1). Thus, we reduce the existence of a solution for the problem (1.1) to the existence of a critical point of $\Phi-\lambda J$ on $H$.

The following estimate will play a key role in the proof of our main results.

Lemma 2.2. For any $x \in H$ and $p>1$, the relation

$$
\max _{k \in \mathbb{Z}(1, T)}\{|x(k)|\} \leq \frac{(T+1)^{(p-1) / p}}{2}\|x\|_{P}
$$

holds.

Proof. Let $\tau \in \mathbb{Z}(1, T)$ such that

$$
|x(\tau)|=\max _{k \in \mathbb{Z}(1, T)}\{|x(k)|\}
$$

Since $x(0)=x(T+1)=0$ for any $x \in H$, by Cauchy-Schwarz inequality, we get

$$
\begin{aligned}
|x(\tau)|=\left|\sum_{k=1}^{\tau} \Delta x(k-1)\right| \leq \sum_{k=1}^{\tau}|\Delta x(k-1)| \leq \tau^{1 / q}\left(\sum_{k=1}^{\tau}|\Delta x(k-1)|^{p}\right)^{1 / p}, \\
|x(\tau)|=\left|\sum_{k=\tau+1}^{T+1} \Delta x(k-1)\right| \leq \sum_{k=\tau+1}^{T+1}|\Delta x(k-1)| \\
\leq(T-\tau+1)^{1 / q}\left(\sum_{k=\tau+1}^{T+1}|\Delta x(k-1)|^{p}\right)^{1 / p}
\end{aligned}
$$

for any $x \in H$, where $q$ is the conjugative number of $p$, that is, $1 / p+1 / q=1$.

If

$$
\sum_{k=1}^{\tau}|\Delta x(k-1)|^{p} \leq \frac{(T+1)^{p-1}}{2^{p} \tau^{p-1}}\|x\|_{P^{\prime}}^{p}
$$

jointly with the estimate (2.12), we get the required relation (2.10).

If, on the contrary,

$$
\sum_{k=1}^{\tau}|\Delta x(k-1)|^{p}>\frac{(T+1)^{p-1}}{2^{p} \tau^{p-1}}\|x\|_{P^{\prime}}^{p}
$$


thus,

$$
\sum_{k=\tau+1}^{T+1}|\Delta x(k-1)|^{p}=\|x\|_{P}^{p}-\sum_{k=1}^{\tau}|\Delta x(k-1)|^{p}<\left(1-\frac{(T+1)^{p-1}}{2^{p} \tau^{p-1}}\right)\|x\|_{P}^{p} .
$$

Combining the above inequality with the estimate (2.13), we have

$$
|x(\tau)|<(T-\tau+1)^{1 / q}\left(1-\frac{(T+1)^{p-1}}{2^{p} \tau^{p-1}}\right)^{1 / p}\|x\|_{P} .
$$

Now, we claim that the inequality

$$
(T-\tau+1)^{1 / q}\left(1-\frac{(T+1)^{p-1}}{2^{p} \tau^{p-1}}\right)^{1 / p} \leq \frac{(T+1)^{(p-1) / p}}{2}
$$

holds, which leads to the required inequality (2.10). In fact, we define a continuous function $v:] 0, T+1[\rightarrow \mathbb{R}$ by

$$
v(s)=\frac{1}{(T-s+1)^{p-1}}+\frac{1}{s^{p-1}} .
$$

This function $v$ can attain its minimum $2^{p} /(T+1)^{p-1}$ at $s=(T+1) / 2$. Since $\tau \in \mathbb{Z}(1, T)$, we have $v(\tau) \geq 2^{p} /(T+1)^{p-1}$, namely,

$$
\frac{2^{p}}{(T+1)^{p-1}} \leq \frac{1}{(T-\tau+1)^{p-1}}+\frac{1}{\tau^{p-1}}
$$

This implies the assertion (2.18). Lemma 2.2 is proved.

\section{Main results}

First, we present our main results as follows.

Theorem 3.1. Let $f(k, \cdot) \in C(\mathbb{R}, \mathbb{R})$ for any $k \in \mathbb{Z}(1, T)$. Put $F(k, \xi)=\int_{0}^{\xi} f(k, s) d s$ for any $\xi \in \mathbb{R}$ and assume that there exist four positive constants $c, d, \mu, \alpha$ with $c<(T+1) / 2^{(p-1) / p} d$ and $\alpha<p$ such that

$\left(\mathrm{A}_{1}\right) \max _{(k, \xi) \in \mathbb{Z}(1, T) \times[-c, c]} F(k, \xi)<\left((2 c)^{p} / T\left[(2 c)^{p}+2(T+1)^{p-1} d^{p}\right]\right) \sum_{k=1}^{T} F(k, d) ;$

$\left(\mathrm{A}_{2}\right) F(k, \xi) \leq \mu\left(1+|\xi|^{\alpha}\right)$.

Furthermore, put

$$
\begin{aligned}
\varphi_{1} & =\frac{p(T+1)^{p-1} T \max _{(k, \xi) \in \mathbb{Z}(1, T) \times[-c, c]} F(k, \xi)}{(2 c)^{p}}, \\
\varphi_{2} & =\frac{p\left[\sum_{k=1}^{T} F(k, d)-T \max _{(k, \xi) \in \mathbb{Z}(1, T) \times[-c, c]} F(k, \xi)\right]}{2 d^{p}},
\end{aligned}
$$


and for each $h>1$,

$$
a=\frac{h(2 c d)^{p}}{2^{p-1} p c^{p} \sum_{k=1}^{T} F(k, d)-T(T+1)^{p-1} p d^{p} \max (k, \xi) \in \mathbb{Z}(1, T) \times[-c, c]}{ }_{(k, \xi)} .
$$

Then, for each

$$
\left.\lambda \in \Lambda_{1}=\right] \frac{1}{\varphi_{2}}, \frac{1}{\varphi_{1}}[
$$

the problem (1.1) admits at least three solutions in $H$ and, moreover, for each $h>1$, there exist an open interval $\Lambda_{2} \subseteq[0, a]$ and a positive real number $\sigma$ such that, for each $\lambda \in \Lambda_{2}$, the problem (1.1) admits at least three solutions in $H$ whose norms in $H$ are less than $\sigma$.

Remark 3.2. By the condition $\left(\mathrm{A}_{1}\right)$, we have

$$
T\left[(2 c)^{p}+2(T+1)^{p-1} d^{p}\right] \max _{(k, \xi) \in \mathbb{Z}(1, T) \times[-c, c]} F(k, \xi)<(2 c)^{p} \sum_{k=1}^{T} F(k, d) .
$$

That is,

$$
2 d^{p}(T+1)^{p-1} T \max _{(k, \xi) \in \mathbb{Z}(1, T) \times[-c, c]} F(k, \xi)<(2 c)^{p}\left[\sum_{k=1}^{T} F(k, d)-T \max _{(k, \xi) \in \mathbb{Z}(1, T) \times[-c, c]} F(k, \xi)\right] .
$$

Thus, we get

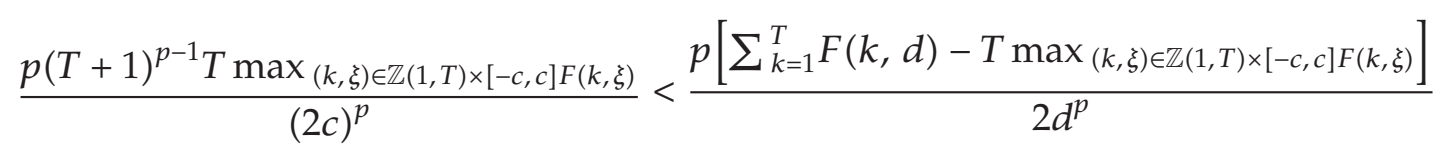

Namely, we obtain the fact that $\varphi_{1}<\varphi_{2}$.

Proof of Theorem 3.1. Let $X$ be the Hilbert space $H$. Thanks to Remark 2.1, we can apply Theorem 1.1 to the two functionals $\Phi$ and $J$. We know from the definitions in (2.5) that $\Phi$ is a nonnegative continuously Gâteaux differentiable and sequentially weakly lower semicontinuous functional whose Gâteaux derivative admits a continuous inverse on $X^{*}$, and $J$ is a continuously Gâteaux differentiable functional whose Gâteaux derivative is compact. Now, put $x_{0}(k)=0$ for any $k \in \mathbb{Z}(0, T+1)$, it is easy to see that $x_{0} \in H$ and $\Phi\left(x_{0}\right)=J\left(x_{0}\right)=0$.

Next, in view of the assumption $\left(\mathrm{A}_{2}\right)$ and the relation (2.4), we know that for any $x \in H$ and $\lambda \geq 0$,

$$
\begin{aligned}
\Phi(x)-\lambda J(x) & =\frac{1}{p} \sum_{k=1}^{T+1}|\Delta x(k-1)|^{p}-\lambda \sum_{k=1}^{T} F(k, x(k)) \\
& \geq \frac{1}{p}\|x\|_{P}^{p}-\lambda \mu \sum_{k=1}^{T}\left(1+|x(k)|^{\alpha}\right) \\
& \geq \sum_{k=1}^{T}\left[\frac{c_{1 p}^{p}}{p}|x(k)|^{p}-\lambda \mu|x(k)|^{\alpha}-\lambda \mu\right] .
\end{aligned}
$$


Taking into account the fact that $\alpha<p$, we obtain, for all $\lambda \in[0,+\infty[$,

$$
\lim _{\|x\| \rightarrow+\infty}(\Phi(x)-\lambda J(x))=+\infty
$$

The condition (i) of Theorem 1.1 is satisfied.

Now, we let

$$
\begin{gathered}
x_{1}(k)= \begin{cases}0, & k=0, \\
d, & k \in \mathbb{Z}(1, T), \\
0, & k=T+1 .\end{cases} \\
r=\frac{(2 c)^{p}}{p(T+1)^{p-1}} .
\end{gathered}
$$

It is clear that $x_{1} \in H$,

$$
\begin{aligned}
& \Phi\left(x_{1}\right)=\frac{1}{p} \sum_{k=1}^{T+1}|\Delta x(k-1)|^{p}=\frac{2 d^{p}}{p} \\
& J\left(x_{1}\right)=\sum_{k=1}^{T} F\left(k, x_{1}(k)\right)=\sum_{k=1}^{T} F(k, d) .
\end{aligned}
$$

In view of $c<((T+1) / 2)^{(p-1) / p} d$, we get

$$
\Phi\left(x_{1}\right)=\frac{2 d^{p}}{p}>\frac{(2 c)^{p}}{p(T+1)^{p-1}}=r .
$$

So, the assumption (ii) of Theorem 1.1 is obtained. Next, we verify that the assumption (iii) of Theorem 1.1 holds. From Lemma 2.2, the estimate $\Phi(x) \leq r$ implies that

$$
|x(k)|^{p} \leq \frac{(T+1)^{p-1}}{2^{p}}\|x\|_{P}^{p}=\frac{p(T+1)^{p-1}}{2^{p}} \Phi(x) \leq \frac{p r(T+1)^{p-1}}{2^{p}}
$$

for any $k \in \mathbb{Z}(1, T)$. From the definition of $r$, it follows that

$$
\left.\left.\Phi^{-1}(]-\infty, r\right]\right) \subseteq\{x \in H:|x(k)| \leq c, \forall k \in \mathbb{Z}(1, T)\} .
$$

Thus, for any $x \in H$, we have

$$
\sup _{x \in \bar{\Phi}^{-1}(]-\infty, r[)}^{w} J(x)=\sup _{\left.\left.x \in \bar{\Phi}^{-1}(]-\infty, r\right]\right)} J(x) \leq T \max _{(k, \xi) \in \mathbb{Z}(1, T) \times[-c, c]} F(k, \xi) .
$$

On the other hand, we get

$$
\frac{r}{r+\Phi\left(x_{1}\right)} J\left(x_{1}\right)=\frac{(2 c)^{p}}{(2 c)^{p}+2(T+1)^{p-1} d^{p}} \sum_{k=1}^{T} F(k, d) .
$$


Therefore, it follows from the assumption $\left(\mathrm{A}_{1}\right)$ that

$$
\sup _{x \in \Phi^{-1}(]-\infty, r[)}^{w} J(x) \leq \frac{r}{r+\Phi\left(x_{1}\right)} J\left(x_{1}\right),
$$

that is, the condition (iii) of Theorem 1.1 is satisfied.

Note that

$$
\begin{aligned}
& \frac{\Phi\left(x_{1}\right)}{J\left(x_{1}\right)-\sup _{x \in \Phi^{-1}(]-\infty, r[)}^{w} J(x)} \\
& \leq \frac{2 d^{p}}{p\left[\sum_{k=1}^{T} F(k, d)-T \max _{(k, \xi) \in \mathbb{Z}(1, T) \times[-c, c]} F(k, \xi)\right]}=\frac{1}{\varphi_{2}},
\end{aligned}
$$

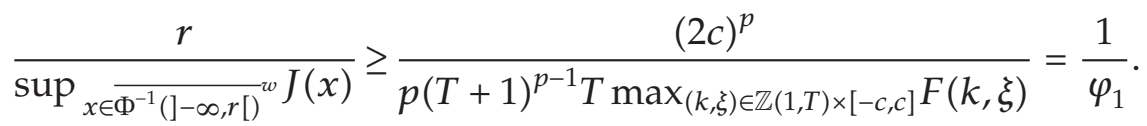

By a simple computation, it follows from the condition $\left(\mathrm{A}_{1}\right)$ that $\varphi_{2}>\varphi_{1}$. Applying Theorem 1.1, for each $\left.\lambda \in \Lambda_{1}=\right] 1 / \varphi_{2}, 1 / \varphi_{1}$, the problem (1.1) admits at least three solutions in $H$.

For each $h>1$, we easily see that

$$
\begin{aligned}
& \frac{h r}{r\left(J\left(x_{1}\right) / \Phi\left(x_{1}\right)\right)-\sup _{x \in \bar{\Phi}^{-1}(]-\infty, r[)}^{w} J(x)} \\
& \quad \leq \frac{h(2 c d)^{p}}{2^{p-1} p c^{p} \sum_{k=1}^{T} F(k, d)-T(T+1)^{p-1} p d^{p} \max _{(k, \xi) \in \mathbb{Z}(1, T) \times[-c, c]} F(k, \xi)}=a .
\end{aligned}
$$

Taking the condition $\left(\mathrm{A}_{1}\right)$ into account, it forces that $a>0$. Then from Theorem 1.1, for each $h>1$, there exist an open interval $\Lambda_{2} \subseteq[0, a]$ and a positive real number $\sigma$, such that, for $\lambda \in \Lambda_{2}$, the problem (1.1) admits at least three solutions in $H$ whose norms in $H$ are less than $\sigma$. The proof of Theorem 3.1is complete.

As a special case of the problem (1.1), we consider the following systems:

$$
\begin{gathered}
\Delta\left[\phi_{p}(\Delta x(k-1))\right]+\lambda w(k) g(x(k))=0, \quad k \in \mathbb{Z}(1, T), \\
x(0)=0=x(T+1),
\end{gathered}
$$

where $w: \mathbb{Z}(1, T) \rightarrow \mathbb{R}$ and $g \in C(\mathbb{R}, \mathbb{R})$ are nonnegative. Define

$$
W(k)=\sum_{t=1}^{k} w(t), \quad G(\xi)=\int_{0}^{\xi} g(s) d s .
$$

Then Theorem 3.1 takes the following simple form. 
Corollary 3.3. Let $w: \mathbb{Z}(1, T) \rightarrow \mathbb{R}$ and $g \in C(\mathbb{R}, \mathbb{R})$ be two nonnegative functions. Assume that there exist four positive constants $c, d, \eta, \alpha$ with $c<(T+1) / 2)^{(p-1) / p} d$ and $\alpha<p$ such that

$\left(\mathrm{A}_{1}^{\prime}\right) \max _{k \in \mathbb{Z}(1, T)} w(k)<\left((2 c)^{p} W(T) / T\left[(2 c)^{p}+2(T+1)^{p-1} d^{p}\right]\right) G(d) / G(c) ;$

$\left(\mathrm{A}_{2}^{\prime}\right) G(\xi) \leq \eta\left(1+|\xi|^{\alpha}\right)$ for any $\xi \in \mathbb{R}$.

Furthermore, put

$$
\begin{gathered}
\varphi_{1}=\frac{p(T+1)^{p-1} T G(c) \max _{k \in \mathbb{Z}(1, T)} w(k)}{(2 c)^{p}}, \\
\varphi_{2}=\frac{p\left[W(T) G(d)-T G(c) \max _{k \in \mathbb{Z}(1, T)} w(k)\right]}{2 d^{p}},
\end{gathered}
$$

and for each $h>1$,

$$
a=\frac{(2 c d)^{p} h}{2^{p-1} p c^{p} W(T) G(d)-p d^{p} T(T+1)^{p-1} G(c) \max _{k \in \mathbb{Z}(1, T} w(k)} .
$$

Then, for each

$$
\left.\lambda \in \Lambda_{1}=\right] \frac{1}{\varphi_{2}}, \frac{1}{\varphi_{1}}[
$$

the problem (3.19) admits at least three solutions in $H$ and, moreover, for each $h>1$, there exist an open interval $\Lambda_{2} \subseteq[0, a]$ and a positive real number $\sigma$ such that, for each $\lambda \in \Lambda_{2}$, the problem (3.19) admits at least three solutions in $H$ whose norms in $H$ are less than $\sigma$.

Proof. Note that from fact $f(k, s)=w(k) g(s)$ for any $k \in \mathbb{Z}(1, T) \times \mathbb{R}$, we have

$$
\max _{(k, \xi) \in \mathbb{Z}(1, T) \times[-c, c]} F(k, \xi)=G(c) \max _{k \in \mathbb{Z}(1, T)} w(k) .
$$

On the other hand, we take $\mu=\eta \max _{k \in \mathbb{Z}(1, T)} w(k)$. Obviously, all assumptions of Theorem 3.1 are satisfied.

To the end of this paper, we give an example to illustrate our main results.

Example 3.4. We consider (1.1) with $f(k, s)=k g(s), T=15, p=3$, where

$$
g(s)= \begin{cases}e^{s}, & s \leq 4 d, \\ s+e^{4 d}-4 d, & s>4 d .\end{cases}
$$

We have that $W(k)=(1 / 2) k(k+1)$ and

$$
G(\xi)= \begin{cases}e^{\xi}-1, & \xi \leq 4 d, \\ \frac{1}{2} \xi^{2}+\left(e^{4 d}-4 d\right) \xi+(1-4 d) e^{4 d}+8 d^{2}-1, & \xi>4 d .\end{cases}
$$

It can be easily shown that, when $c=1, d=15, \eta=e^{60}$, and $\alpha=2$, all conditions of Corollary 3.3 are satisfied. 


\section{Acknowledgments}

This work is supported by the National Natural Science Foundation of China (no. 10571032) and Doctor Scientific Research Fund of Jishou university (no. jsdxskyzz200704).

\section{References}

[1] R. Avery and J. Henderson, "Existence of three positive pseudo-symmetric solutions for a one dimensional discrete p-Laplacian," Journal of Difference Equations and Applications, vol. 10, no. 6, pp. 529-539, 2004.

[2] Z. He, "On the existence of positive solutions of $p$-Laplacian difference equations," Journal of Computational and Applied Mathematics, vol. 161, no. 1, pp. 193-201, 2003.

[3] Y. Li and L. Lu, "Existence of positive solutions of $p$-Laplacian difference equations," Applied Mathematics Letters, vol. 19, no. 10, pp. 1019-1023, 2006.

[4] Y. Liu and W. Ge, “Twin positive solutions of boundary value problems for finite difference equations with $p$-Laplacian operator," Journal of Mathematical Analysis and Applications, vol. 278, no. 2, pp. 551$561,2003$.

[5] R. P. Agarwal, K. Perera, and D. O'Regan, "Multiple positive solutions of singular and nonsingular discrete problems via variational methods," Nonlinear Analysis: Theory, Methods \& Applications, vol. 58, no. 1-2, pp. 69-73, 2004.

[6] Z. M. Guo and J. S. Yu, "Existence of periodic solutions and subharmonic solutions on second order superlinear difference equations," Science in China Series A, vol. 33, no. 3, pp. 226-235, 2003.

[7] L. Jiang and Z. Zhou, "Existence of nontrivial solutions for discrete nonlinear two point boundary value problems," Applied Mathematics and Computation, vol. 180, no. 1, pp. 318-329, 2006.

[8] Z. Zhou, J. Yu, and Z. Guo, "Periodic solutions of higher-dimensional discrete systems," Proceedings of the Royal Society of Edinburgh: Section A, vol. 134, no. 5, pp. 1013-1022, 2004.

[9] Z. Zhou, J. Yu, and Z. Guo, "The existence of periodic and subharmonic solutions to subquadratic discrete Hamiltonian systems," The ANZIAM Journal, vol. 47, no. 1, pp. 89-102, 2005.

[10] B. Ricceri, "On a three critical points theorem," Archiv der Mathematik, vol. 75, no. 3, pp. 220-226, 2000.

[11] G. Bonanno, "A critical points theorem and nonlinear differential problems," Journal of Global Optimization, vol. 28, no. 3-4, pp. 249-258, 2004. 